\title{
Elongated Mandibular Premolar: A New Morphological Variant
}

\author{
Heather J. H. Edgar* and Paul W. Sciulli** \\ ${ }^{*}$ Maxwell Museum of Anthropology, University of New Mexico, Albuquerque, New Mexico \\ **Department of Anthropology, The Ohio State University, Columbus, Ohio
}

\begin{abstract}
A previously unreported morphological variant, elongated premolar, is described and analyzed. The elongated premolar is mandibular and may affect the anterior or posterior premolar in the field. It appears phenotypically to be more rectangular (with the long axis mesiodistal) than 'normal' premolars. Dentitions of European Americans, African Americans, and an admixed group of Native African/European Americans were examined for the presence of this characteristic. Elongated premolars were found in 19 teeth in the 458 individuals included in the study. Mesiodistal diameter,
\end{abstract}

During data collection for a larger study (Edgar, 2002), it was noted that some mandibular premolars were more rectangular than 'normal' premolars. The affected teeth appear to be either compressed in the buccolingual dimension or longer in the mesiodistal dimension. The affected premolar is thus more rectangular or elliptical than hexagonal or rounded, like most mandibular premolars. Additionally, the cusps of the affected premolars appear closer together, and somewhat angled toward each other in some cases. Although affected posterior mandibular premolars were noted, more anterior premolars were seen with this condition.

\section{MATERIALS}

A total of 458 individual dentitions were examined for the presence of affected premolars. A list of the samples from which these individuals were drawn, their ancestry, and the frequency of trait presence is listed in Table 1. In these dentitions 19 elongated mandibular premolars were noted, 14 anterior and five posterior.

\section{METHODS}

Measurements were made of 14 affected and 22 non-affected anterior premolars. All individuals are represented by the following three measurements:

Mesiodistal diameter (MD): This measure was the greatest length of the tooth in the mesiodistal plane. Adjustments were made if the tooth was rotated out of 'normal' occlusion (Goose, 1963; Hillson, 1986, 1996; Moorrees, 1957; Moorrees et al., 1957). Teeth with excessive wear were excluded from the study (Keiser, 1990). buccolingual diameter, and cusp distance were measured for 14 affected and 22 unaffected anterior premolars. Principal components analysis shows that elongated and non-elongated premolars differ primarily in shape and not size, with elongated premolars attaining their overall shape due primarily to an increase in the mesiodistal dimension. Thus, the suggested description of this feature is elongated premolar (referring to the mesiodistal dimension), rather than compressed premolar (referring to the buccolingual dimension). Dental Anthropology 2004;17(1):24-27.

Buccolingual diameter (BL): the maximum diameter of the tooth crown perpendicular to the MD measurement (Goose, 63; Hillson, 1986, 1996; Moorrees, 1957; Moorrees et al. , 1957).

Cusp Distance (CD): This is a measure of the distance between apices of the two main cusps of the premolar, one buccal and one lingual. If more than one lingual cusp was present, the largest cusp was the one included in the measurement. If there was no lingual cusp, the measure was taken from the apex of the buccal cusp to the center of the lingual marginal ridge.

In order to investigate the nature of the size and shape differences between the affected and non-affected premolars, we performed principal components analysis (PCA) on the logarithmically transformed $\mathrm{BL}$, $\mathrm{MD}$, and $\mathrm{CD}$ dimensions. We used this approach as it allows the analysis of variation in the premolars to include multiple measures, while accounting for the interaction among the measures. Noting the pattern of these relationships often provides insights into the relationships between the size and shape of features as well as how size and shape interact in the development of the feature (Jolicoeur, 1963).

PCA was performed with SAS software (SAS Statistical Institute, 2003). The calculation of the principal components of a covariance matrix yields the direction cosines and lengths of the principal axes of

\footnotetext{
*Address for correspondence: Heather H. J. Edgar, Maxwell Museum of Anthropology, MSC01 1050, 1 University of New Mexico, Albuquerque, New Mexico 87131-0001 E-mail: hjhedgar@unm.edu
} 
the multidimensional scatter of points. This will show which combination of features is most variable and uncorrelated with each other. The eigenvector matrix contains the direction cosines of the principal axes. If all of the direction cosines of an eigenvector are of the same sign, this axis represents a simultaneous increase (or decrease) in all of the features, and is usually considered to represent size variation. If, on the other hand, the signs of the direction cosines differ, then some features increase while others decrease. Axes of this sort are often considered to reflect shape variation, as this represents an increase in some dimensions and a simultaneous decrease in others.

In addition to differences in sign, the direction cosines may differ in magnitude. For example, if a feature increased in such a way that the proportions of all dimensions remained constant, then the relations among the dimensions would be isometric. This situation will be reflected in the direction cosines of an eigenvector when they all have the same sign and magnitude equal to $1 / \sqrt{ } \mathrm{p}$, where $\mathrm{p}$ is the number of variables.

\section{RESULTS}

Figures 1 and 2 are affected anterior and posterior premolars, respectively. If every dentition examined in this study contained a full complement of mandibular premolars, 1,832 teeth would have been examined, and the trait frequency of expression would be $1.09 \%$. However, a minority of observed dentitions was incomplete, so the actual frequency of the trait expression is somewhat higher. Out of the 458 dentitions observed, 14 had at least one affected tooth,

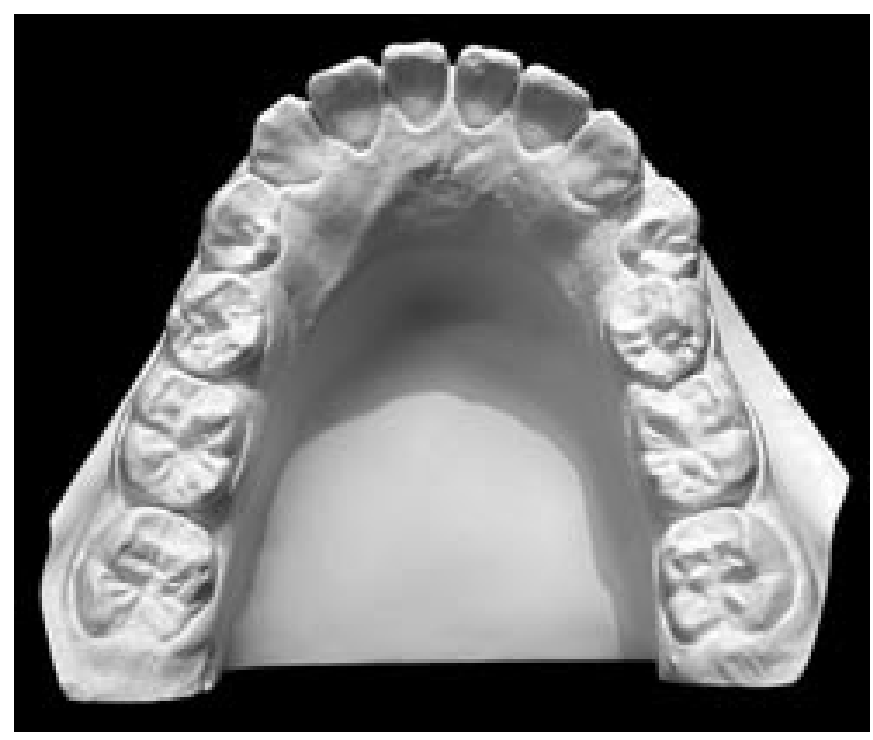

Fig 1. Elongated anterior premolars. Bilaterally, the anterior premolars exhibit a marked reduction in size of the buccolingual dimension, but no loss of mesiodistal length. giving a sample frequency of $3.06 \%$. Of these 14 , seven individuals showed unilateral expression in an anterior premolar, four showed unilateral expression in a posterior premolar, two showed bilateral expression in the anterior premolars, and one showed expression in both one anterior and one posterior premolars.

Table 2 contains the results of the PCA of the affected and unaffected premolar samples. In the unaffected sample the direction cosines of the first axis are all the same sign, indicating this axis represents size variation. This observed first eigenvector differs from hypothetical isometry (0.5774 0.57740 .5774$)$ by only $45^{\circ}$, indicating that the proportions of all dimensions are constant. The second and third axes have direction cosines of differing signs indicating that they represent shape variation. The second axis shows direction cosines for the BL and MD dimensions of the same sign and approximate magnitude, while the direction cosine corresponding to the $\mathrm{CD}$ dimension is about 2.5 times smaller. The third axis from the PCA of the unaffected sample represents a contrast between the BL and MD dimensions, with the former increasing while the latter decreases.

In the affected premolar sample, the direction cosines of the first axis are all of the same sign. This axis, like the first axis of the unaffected premolar sample, represents size variation. However, unlike the first axis of the unaffected premolar sample, the first axis of the affected premolar sample does not appear to represent isometry. The direction cosine of the MD diameter is about 1.5 times smaller than those of the BL and $\mathrm{CD}$ dimensions, and the eigenvector differs from hypothetical isometry by $14.6^{\circ}$. The second and third axes of the affected premolar sample, like the sample of unaffected premolars, contain direction cosines with different signs, indicating these two axes represent

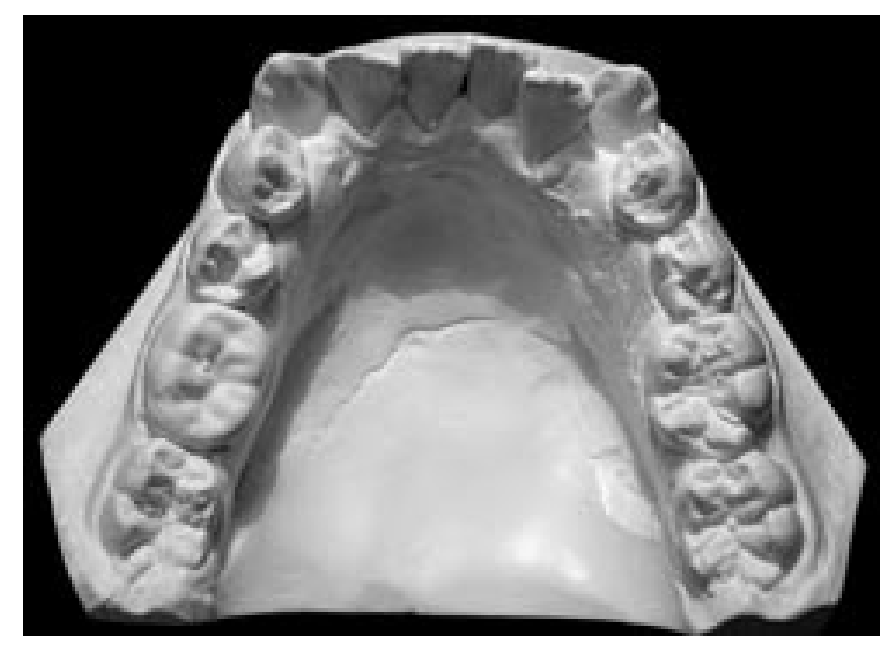

Fig 2. Elongated posterior premolars. The two posterior premolars, but notably the tooth on the right, is compressed buccolingually, but no loss of mesiodistal length. 
TABLE 1. Sample materials.

\begin{tabular}{|c|c|c|c|c|c|}
\hline \multirow[b]{2}{*}{ Group } & \multirow[b]{2}{*}{ Ancestry } & \multirow[b]{2}{*}{ Reference } & \multirow[b]{2}{*}{$\mathrm{N}$} & \multicolumn{2}{|c|}{ Affected } \\
\hline & & & & Anterior & Posterior \\
\hline New York Twins & European American & Osborne et al., 1958 & 102 & 4 & 1 \\
\hline U T Memphis & European American & Lease and Harris, 2001; & 101 & & \\
\hline & Afrionn Amoricon & Edgar, 2002 & 101 & 0 & 0 \\
\hline Gullah & African American & Menegaz-Bock, 1968; & & 1 & 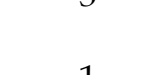 \\
\hline Hollister-Haliwa & $\begin{array}{l}\text { Native American } \\
\text { African American } \\
\text { European American }\end{array}$ & Menegaz-Bock, 1968 & 100 & 0 & 0 \\
\hline & & Totals & 458 & 14 & 5 \\
\hline
\end{tabular}

shape variation. Both the second and third axes show a large direction cosine for the MD dimension in relation to the $\mathrm{BL}$ dimension. Compared to the sample of unaffected premolars the MD dimension on the second and third axes of the affected premolars represents a much larger relative increase. The direction cosine for the $C D$ dimension on the second and third axes have the same sign as in the sample of unaffected premolars, but with a smaller magnitude in the second axis and a greater magnitude in the third axis.

\section{CONCLUSIONS}

In the PCA results for both unaffected and affected premolars, the first axis represents size variation. The measures of the unaffected premolars are isometric on the first axis, but the affected premolars deviate from isometry, with the $\mathrm{BL}$ dimension being larger that the $\mathrm{MD}$. This result is not expected, as the affected premolars appear to be narrower in the BL direction. However, the first axis of the affected premolar may simply reflect the general size component of premolars in which increase in the BL dimension is fairly constant.

The second and third axes represent shape variation in both premolar samples, and it is in these axes that the overall phenotypic differences are manifest. For the unaffected premolar sample, in the second axis, the BL and MD direction cosines are approximately equal and the $\mathrm{CD}$ direction cosine is larger in magnitude but of opposite sign. In the third axis the direction cosine of the BL dimension is greater than that of the MD dimension, and the $\mathrm{CD}$ dimension direction cosine is negligible. These results describe a 'normal' premolar that is generally oval in outline, with the BL dimension slightly

TABLE 2. Eigenvector matrices, percent of total variation accounted for by the eigenvalues, and mean vectors from PCA of logrithmically transformed BL, MD, and CD dimensions.

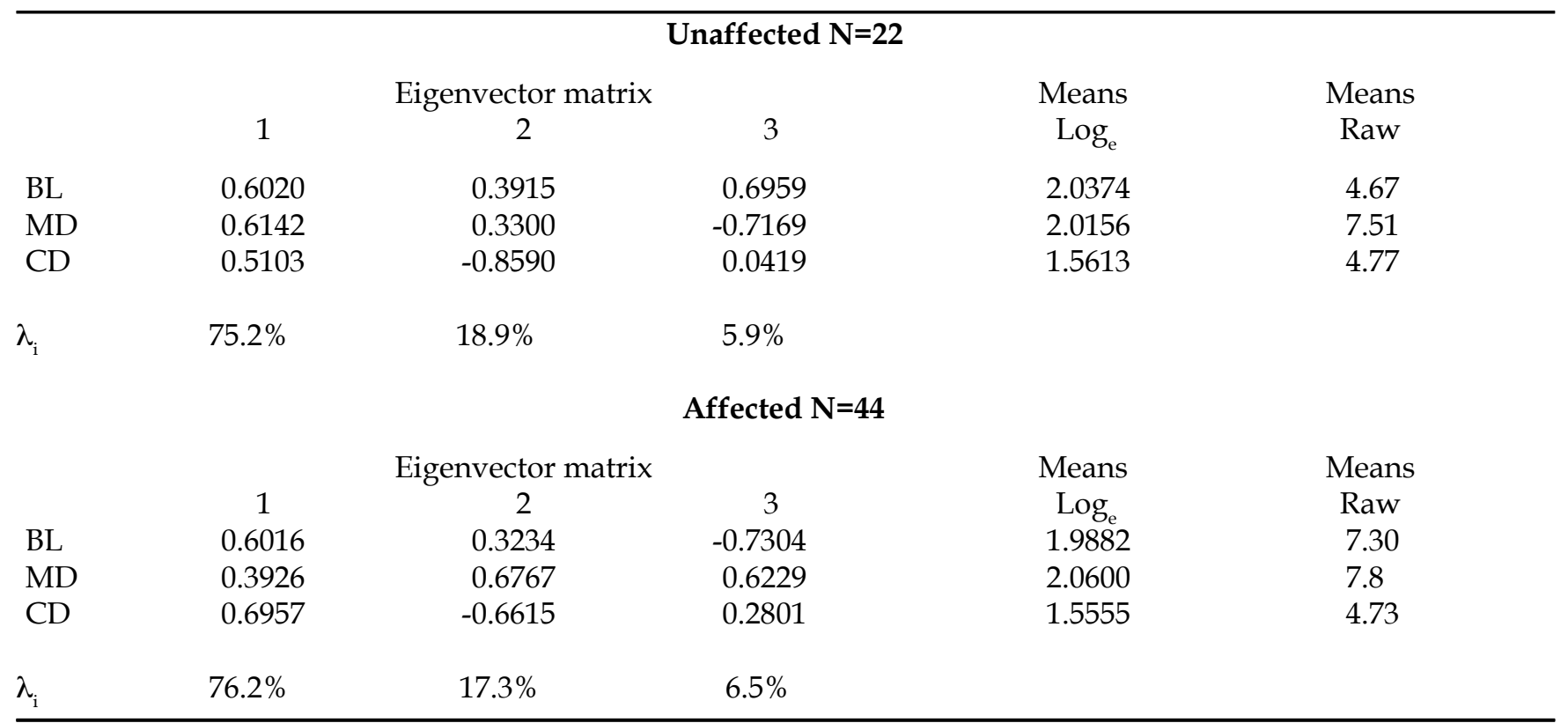


larger than the MD.

For the affected premolar sample, the direction cosine for the MD dimension is somewhat larger than the BL direction cosine in the second axis, and much larger than the $\mathrm{BL}$ direction cosine in the third axis. The direction cosine of the CD dimension is not as small in the second axis as it is in the unaffected sample, and it is larger in the third axis than in the 'normal' sample. This pattern of interaction describes a tooth that is primarily elongated in the MD direction, more rectangular than 'normal' premolars, and with cusps somewhat closer together.

It should be noted that elongated premolars are detectable as a morphological variant. It is not necessary to perform metric analysis to include an observation of its presence or absence in a morphological analysis of a dentition. The metric analysis described here was performed simply to determine if the final phenotype was the result of a predominant reduction in BL growth or and extension of MD growth. We are interested to hear from other researchers who may have noted this characteristic and/or have data concerning its expression and frequency.

\section{LITERATURE CITED}

Edgar HJH. 2002. Biological Distance and the African American Dentition. Ph.D. dissertation, Ohio State University.

Goose DH. 1963. Dental measurement: an assessment of its value in anthropological studies. In: Brothwell DR, editor. Dental anthropology. London: Pergamon Press, p 263-270.

Hillson SW. 1986. Teeth. Cambridge: Cambridge University Press.

Hillson SW. 1996. Dental anthropology. Cambridge: Cambridge University Press.

Jolicoeur P. 1963. The multivariate generalization of the allometry equation. Biometrics 19:497-499.

Keiser JA. 1990. Human adult odontometrics. Cambridge: Cambridge University Press.

Lease LR, Harris EF. 2001. Absence of association between body size and deciduous tooth size in American Black children. Dental Anthropology 15: 7-12.

Menegaz-Bock RM. 1968. An Investigation of the Genetic Basis for Structural Relationships in the Anterior Dentition. Ph.D. dissertation, University of Chicago.

Moorrees CFA. 1957. The Aleut dentition. Cambridge: Harvard University Press.

Moorrees CFA, Thomsen SO, Jensen E, Yen PKJ. 1957. Mesiodistal crown diameters of deciduous and permanent teeth. J Dent Res 36:39-47.

Osborne RH, Horowitz SL, de George FV. 1958. Genetic variation of tooth dimensions: a twin study of the permanent anterior teeth. Am J Hum Gen 10:350356.

SAS Statistical Institute Inc., 2003.

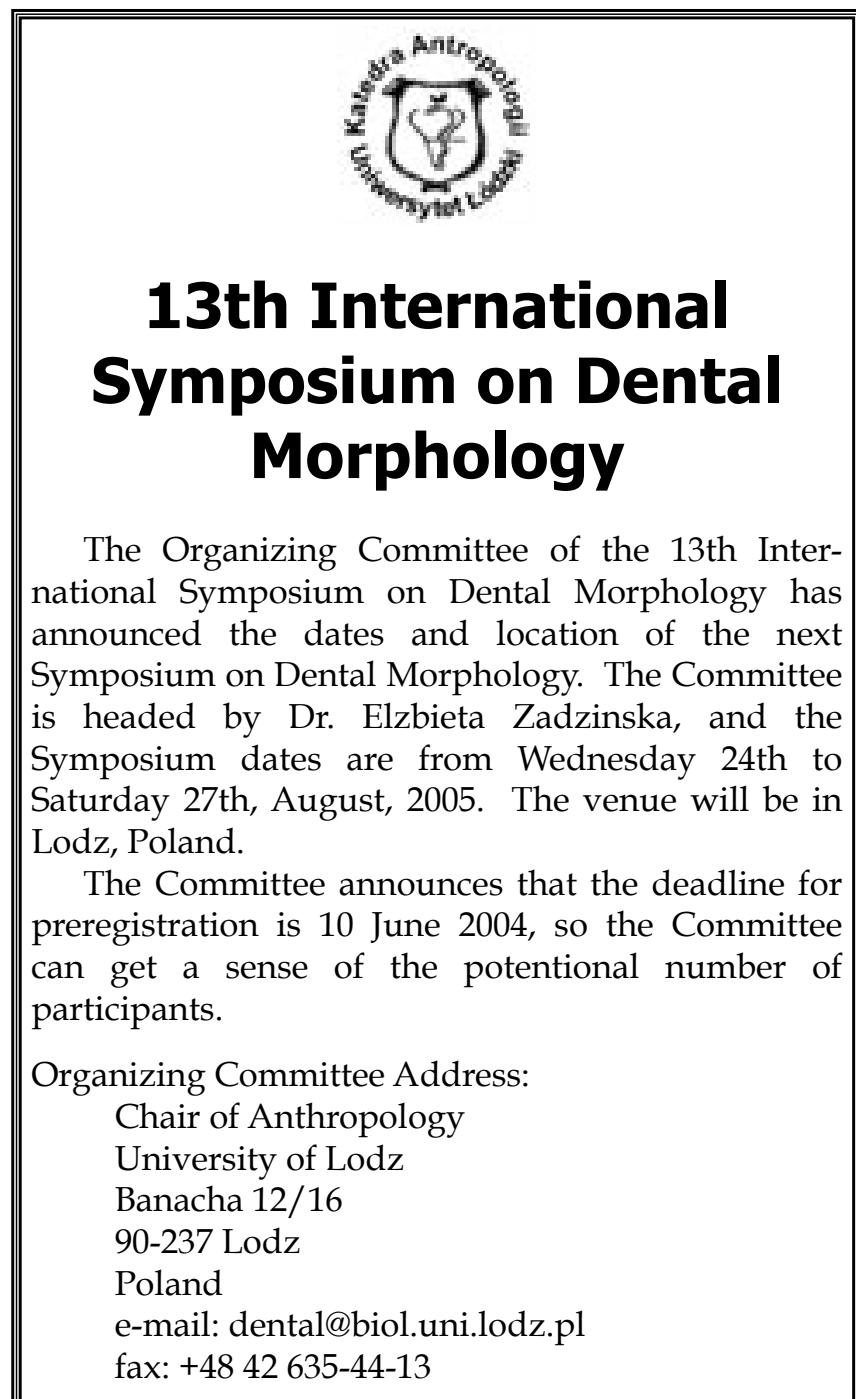

More information will be supplied as it becomes available from the Organizing Committee

The Editor

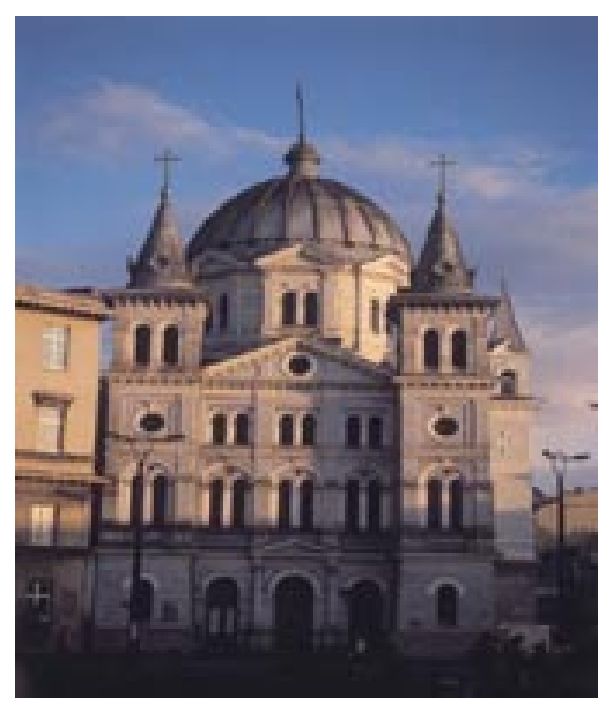

The Cathedral in Lodz 